\title{
Experiencias del cuidado de enfermería en contexto intercultural
}

\author{
Experiences of nursing care in intercultural context
}

\section{Experiências de enfermagem de enfermagem em contexto intercultural}

\author{
Deibys Carrasquilla Baza1 Celenny Pérez Quintero² \\ ${ }^{1}$ Doctor en Ciencias Políticas, antropólogo. Profesor Universidad Cooperativa de Colombia, sede \\ Santa Marta. Correo electrónico: deibys.carrasquillab@campusucc.edu.co \\ ${ }^{2}$ Doctora en Ciencias Gerenciales, enfermera. Profesora Universidad Cooperativa de Colombia, sede \\ Santa Marta. Correo electrónico: celenny.perezq@campusucc.edu.co \\ Cómo citar este artículo en edición digital: Carrasquilla Baza, D., \& Pérez Quintero, C. (2018). \\ Experiencias del cuidado de enfermería en contexto intercultural. Cultura de los Cuidados (Edición \\ digital), 22(51). Recuperado de <http://dx.doi.org/10.14198/cuid.2018.51.14> \\ Correspondencia: Mz 67 Casa 2. Barrio El Pando. Santa Marta - Colombia. \\ Correo electrónico: deibys.carrasquillab@campusucc.edu.co \\ Recibido: 28/10/2017; Aceptado: 11/03/2018.
}

\begin{abstract}
Objective: to understand the experiences of nursing care for indigenous patients in an intercultural context. Materials and methods: qualitative study, guided by the ethnographic method, combined documentary review, observation and ethnographic interview carried out with 26 people, 12 nurses, 3 social workers, 1 manager of an institution providing indigenous health services and 10 indigenous people. Results: the experiences were evidenced from the representations about the care of the indigenous patient, generally seen as a difficult situation, due to their pathologies, hygiene and rejection of the care actions. Communication was another of the aspects that marked the experience, due to the difficulty of handling different languages. The strategies and interventions were another determining factor of them, mainly their empirical development, far from the theoretical intercultural nursing support. Conclusion: there is a need to advance in the overcoming of ethnocentric practices for
\end{abstract}

the improvement of the quality and humanization of nursing care in an intercultural context.

Keywords: Nursing care, indigenous patient, hospital institutions, qualitative research

\section{RESUMEN}

Objetivo: comprender las experiencias de cuidado de enfermería a pacientes indígenas en contexto intercultural. Materiales y métodos: estudio cualitativo, orientado desde el método etnográfico, combinó revisión documental, observación y entrevista etnográfica realizada a 26 personas: 12 enfermeras, 3 trabajadoras sociales, 1 gerente de una institución prestadora de servicios de salud indígena y 10 indígenas. Resultados: las experiencias se evidenciaron desde las representaciones sobre el cuidado al paciente indígena, visto generalmente como una situación difícil, debido a sus patologías, higiene y rechazo a las acciones de cuidado. La comunicación fue otro de los aspectos que marcaron la experiencia, debido a la dificultad 
por el manejo de lenguas diferentes. Las estrategias e intervenciones fue otro factor determinante de ellas, principalmente su desarrollo empírico, alejado del soporte teórico intercultural enfermero. Conclusión: existe la necesidad de avanzar en la superación de prácticas etnocéntricas para el mejoramiento de la calidad y humanización del cuidado de enfermería en contexto intercultural.

Palabras clave: Cuidado de enfermería, paciente indígena, instituciones hospitalarias, investigación cualitativa

\section{RESUMO}

Objetivo: compreender as experiências de cuidados de enfermagem para pacientes indígenas em um contexto intercultural. Materiais e métodos: estudo qualitativo, orientado pelo método etnográfico, revisão documental combinada, observação e entrevista etnográfica realizada com 26 pessoas, 12 enfermeiras, 3 assistentes sociais, 1 gerente de IPSI e 10 indígenas. Resultados: as experiências foram evidenciadas a partir das representações sobre o cuidado do paciente indígena, geralmente visto como uma situação difícil, devido a suas patologias, higiene e rejeição das ações de cuidados. A comunicação foi outro dos aspectos que marcaram a experiência, devido à dificuldade de lidar com diferentes idiomas. As estratégias e intervenções foram outro fator determinante delas, principalmente seu desenvolvimento empírico, longe do suporte teórico de enfermagem intercultural. Conclusão: é necessário avançar na superação de práticas etnocêntricas para melhorar a qualidade e a humanização dos cuidados de enfermagem em um contexto intercultural.

Palavras-chave: Cuidados de enfermagem, paciente indígena, instituições hospitalares, pesquisa qualitativa

\section{INTRODUCCIÓN}

El desarrollo epistémico enfermero ha avanzado lo suficiente para lograr problematizar $\mathrm{y}$ generar propuestas que tienen en cuenta el papel de la cultura en los procesos relacionados con el cuidado de enfermería, tal como se advierte en los enfoques enfermería transcultural (Leininger \& McFarland, 2002; McFarland,
2011), antropología de los cuidados (Siles González et al., 2001, 2001), enfermería antropológica (Casassa García, Ramírez, Sánchez, \& Campos, 2010; Poveda, 2001), competencia cultural (Lillo Crespo, Vizcaya Moreno, Santamaría, Mario, \& Galao Malo, 2004; Purnell, 1999), entre otros. Sin embargo, las condiciones en las cuales se desarrolla la profesión, así como las variaciones de la práctica de enfermería en diferentes lugares del mundo, ameritan una revisión de la situación en la que se encuentra el cuidado de enfermería en contextos de diversidad cultural.

La importancia y vigencia de la temática se encuentra tanto en el campo asistencial como el investigativo. Para el primero, en el sentido de una revisión constante del estado de la práctica de enfermería, en este caso, relacionado con la manera en que se está considerando la cultura en el cuidado que se está brindando, pero también por su asociación al cuidado humanizado, debido al carácter holístico con el que se encuentra asociado este concepto. En el caso de la investigación en enfermería, significa el grado de articulación de este campo con el primero, en el sentido del aporte del conocimiento en el mejoramiento de la práctica clínica en contexto de diversidad cultural.

En las últimas tres décadas, con la creación de la enfermería transcultural, la relación de la enfermería - antropología y en general por el interés de la primera por el papel de la cultura en los cuidados, el número de enfoques dedicados al tema, así como los estudios realizados han venido en aumento. Sin embargo, la mayoría de estos estudios se desarrollan desde revisiones sistemáticas y artículos de reflexión, siendo poco el material etnográfico que posee la enfermería como base de su conocimiento sobre la diversidad cultural y del estado de desarrollo de su práctica. En algunos intentos, se evidencia el poco manejo del concepto de cultura, así como las habilidades metodológicas para encarar la profundidad y complejidad de la dinámica cultural de las poblaciones humanas. Gran parte de estos trabajos se extienden en definiciones y teorías sobre la cultura, pero difícilmente pueden captar lo que este concepto significa. Situación similar ocurre con la noción de lo intercultural, limitada a su definición literal de entre culturas, que muchas veces oculta la dinámica de 
poder desigual manifiesta entre la cultura mayoritaria o dominante y las que son minoría, y en ocasiones subalternas. El mundo enfermero y de la salud en general no escapa de estas dinámicas, por lo que es necesario valorar el estado en el que se encuentra la práctica asistencial en estos contextos, comprender las prácticas y procesos que la sustentan y luego plantear una mirada crítica dirigida a intervenir los factores que imposibilitan el cuidado desde los parámetros científicos y humanísticos que promueve la enfermería. En este sentido, el objetivo de este artículo es comprender las experiencias de cuidado de enfermería a pacientes indígenas en contexto intercultural.

\section{MATERIALES Y MÉTODOS}

Se realizó un estudio cualitativo con enfoque etnográfico, para el cual fueron tenidas en cuenta las principales recomendaciones que para este tipo de estudios ofrece la antropología, principalmente los planteamientos de Hammersley y Atkinson (2001) y Guber (2011). De esta última, se tomaron orientaciones generales y sus aportes en torno a la concepción de la etnografía como enfoque, método y escritura; también el entendimiento de esta como el paso del desconocimiento - conocimiento reconocimiento, como experiencia guía de los procesos cognitivos. Los primeros, por su parte, constituyeron el referente frente a los procedimientos implementados, que definieron en gran parte el uso de las técnicas de recolección de la información.

Las técnicas implementadas fueron coherentes a la concepción clásica de la etnografía: observación y entrevista. La observación fue no participante y se limitó a períodos cortos, en tres centros de salud de la ciudad, principalmente en la zona de espera de urgencias y consulta general, así como en los servicios donde se permitió el acceso. Esta técnica fue muy limitada para observar la situación de los indígenas, por la dificultad en el conocimiento de la lengua, la disposición de estas personas a comunicarse en castellano y por la situación que los llevó a ese lugar: la enfermedad. En el caso de la labor enfermera se realizó el intento, pero la frecuencia de atención a indígenas no es tan amplia para que coincidiera con el período de observación. Por estos motivos, el estudio se concentró en la entrevista.
La observación realizada fue útil para establecer los participantes del estudio, principalmente de enfermería. Para esto se utilizó la bola de nieve, aprovechando los protocolos institucionales y la gestión que permite la autorización de realización de la investigación. En ese sentido, las personas responsables indicaron nombres del personal con mayor experiencia en el cuidado a indígenas, de acuerdo al tiempo de trabajo, como el servicio presentado. Esta información fue corroborada a partir de los datos de atención a comunidades indígenas, verificando los servicios en los que más se atienden estos pacientes según su patología. A su vez, en contacto directo con estas personas, indicaron los nombres de enfermeras con experiencia en la temática. El caso de los indígenas fue diferente, siendo dos los tipos de personas elegidas: aquellas que recibieron atención en los últimos dos años y personas que siendo de la comunidad, hayan tenido contacto con la atención en salud, de las cuales resultaron una auxiliar de enfermería y una enfermera.

La modalidad elegida para la entrevista fue la etnográfica o en profundidad, siguiendo a los autores ya mencionados. En ese sentido, la entrevista fue tomada como observación, en el sentido de la reconstrucción de la realidad que permiten los testimonios recopilados. Asimismo, se trató de entrevistas semiestructuradas e informales, orientadas por temáticas generales o preguntas generales que motivaran el desarrollo de un diálogo. Las personas entrevistadas fueron 26, de las cuales 12 fueron enfermeras, un gerente de una Institución Prestadora de Servicios de Salud, 3 trabajadoras sociales y 10 indígenas (de las comunidades kogui, arhuaco, wiwa y chimila) Las entrevistas tuvieron una duración aproximada de 50 minutos, fueron grabadas y posteriormente trascritas. El análisis de la información se realizó a partir de la codificación y uso de matriz de análisis de categorías, de acuerdo a la forma en que fueron emergiendo los códigos y su relación con las categorías. En ese sentido, las categorías fueron representaciones sobre el paciente indígena, relación enfermera paciente, comunicación, momentos del cuidado y rechazo a los procedimientos. 
Para el desarrollo del estudio se tuvieron en cuenta los aspectos éticos emanados en la Declaración de Helsinki, así como en la legislación colombiana mediante la Ley 266 de 1996 y la resolución 8430 de 1993, garantizando los criterios de confidencialidad y autonomía mediante el consentimiento informado.

\section{RESULTADOS}

\section{Contexto intercultural}

El estudio se realizó en Santa Marta, una ciudad ubicada al norte de Colombia, de la cual se resalta su historia, geografía y diversidad cultural. Es la más antigua de Colombia (fundada en 1525) y se encuentra entre el Mar Caribe, la Sierra Nevada de Santa Marta (SNSM) y la Ciénaga Grande, tres complejos ecosistemas que han marcado los procesos de desarrollo de la vida humana en estos lugares del planeta, desde el período prehispánico, la colonización y la vida cotidiana actual. Debido a estas características, se ha configurado en ella una población heterogénea que incluye tanto la vida rural como la urbana, generalmente influenciadas mutuamente, así como la presencia de comunidades indígenas que la habitan (kogui, wiwas, arhuacos, chimilas) y la visitan (wayuu, yukpa), todos ellos sobrevivientes de la colonización luego de haberse refugiado a las zonas de difícil acceso donde viven actualmente. Incluye también población afrodescendiente, migrantes o desplazados y extranjeros que han llegado atraídos por el turismo, y que incluyen un matiz más al crisol multicultural de la ciudad.

Este panorama le ha permitido la consolidación de una amplia dinámica cultural, que invita a indagar sobre las condiciones que tiene la ciudad y el sector salud para enfrentar la demanda de atención en salud intercultural, para lo cual este estudio se concentra en las experiencias del personal de enfermería, frente al cuidado brindado a la población indígena. Como parte de la indagación realizada, se encontró que, aspectos como la representación de las enfermeras sobre el paciente indígena, la comunicación, estrategias de abordaje e intervenciones, constituyeron los principales derroteros que contribuyen a revelar la dinámica de la experiencia enfermera, principalmente en términos de la posibilidad de brindar un cuidado congruente con la cultura.

Esta dinámica del cuidado enfermero a pacientes indígenas se ha consolidado a través de las últimas dos décadas, alcanzando cambios importantes. Inicialmente, las instituciones del Estado fundamentaban la política de atención en salud en brigadas y programas en los que el personal de la salud, conformado por un equipo no indígena, incursionaba en zonas rurales de difícil acceso y llevaba servicios de salud occidental, con poco conocimiento de la cultura. Sin embargo, a partir de la ley 100 de 1993 y la gestión de los líderes, los indígenas lograron crear sus Entidades Promotoras de Salud Indígenas (EPSI) e Instituciones Prestadoras de Servicios de Salud Indígena (IPSI), mientras el Estado colombiano, poco a poco reconocía la necesidad de incluir la perspectiva cultural, como se logró consolidar con el Sistema Indígena de Salud Propia Intercultural (SISPI) y el enfoque diferencial que reconoce la política pública en salud actual (Plan Decenal de Salud).

Con la ley 100 y las modificaciones realizadas por la ley estatutaria en salud (que rige el sistema de salud colombiano) y el funcionamiento de las EPSI e IPSI, la dinámica de la atención en salud a la población indígena cambió, aumentando el número de usuarios en el sector salud de la ciudad. Durante ese período (aproximadamente dos décadas), la experiencia de cuidado de enfermería a pacientes indígenas ha aumentado, alcanzando procesos a los cuales merece hacerles seguimiento con el fin de identificar el estado en el que se encuentra, así como señalar directrices sobre sus avances y aspectos críticos, que permitan mejorar su ejecución en el futuro.

\section{Representaciones enfermeras sobre el paciente indígena}

La representación se refiere a la producción del sentido a través del lenguaje o en otras palabras, a las prácticas de producción de significado (Hall, 2010), en este caso, sobre cómo el personal de enfermería ha construido el significado sobre el paciente indígena, durante la experiencia de cuidado en un contexto intercultural como el que ofrece Santa Marta. En este sentido, la representación de la enfermera 
sobre el indígena es determinante en la comprensión de la experiencia de cuidado en el contexto intercultural, porque implica entender una serie de elementos vitales, como qué tanto sabe el partícipe de una cultura (occidental) de la otra (indígena) o hasta qué punto las valoraciones y juzgamientos que realiza los puede estar realizando desde parámetros que ha establecido la cultura de la que hace parte y, por lo tanto, caer en prácticas etnocéntricas. De igual forma, la representación obedece a una dinámica histórica y bajo las pautas, códigos y significado que una sociedad le otorga. En el caso de Santa Marta, Colombia y en general el Caribe y América Latina, las representaciones sobre lo indígena han estado insertas bajo regímenes de representación instaurados durante la experiencia colonial, la cual, clasificó a las poblaciones a partir de sus características étnico - raciales, de género y clase, estableciendo jerarquías, entre las cuales, lo indígena y lo negro fueron ubicados en el peldaño más bajo (Quijano, 2014; Sarrazin, 2015).

Las representaciones construidas por el personal de enfermería sobre el paciente indígena son heterogéneas e insertas en un contexto complejo determinado por el quehacer cotidiano de la enfermería, así como las personas que la practican. Sin embargo, se advierte una serie de tendencias que marcan la dinámica del cuidado de enfermería brindado a comunidades indígenas, muchos de los cuales, aparecen como aspectos determinantes de su desarrollo. Estas representaciones oscilan entre aspectos generales de la población indígena, las patologías más frecuentes de la población, aspectos de su higiene y su actitud frente a las acciones de cuidado. Cuando se referían a la experiencia de cuidado de pacientes indígenas, la mayor parte del personal de enfermería la describían como una "situación difícil”, principalmente por las implicaciones de la práctica profesional en contexto intercultural. Esta dificultad, por tanto, parte del choque cultural, pero sobre todo de la poca competencia para la interacción con un paciente de una cultura distinta. También corresponde al efecto de la representación que tienen sobre ese tipo de pacientes. En cuanto a las patologías más frecuentes, se representa al paciente indígena relacionados con la desnutrición, enfermedades infectocontagiosas como la tuberculosis $\mathrm{y}$ otras del tipo gastrointestinal. Es poco frecuente relacionarlos con enfermedades crónicas, aunque reconocen que estas últimas han venido en aumento en los últimos años.

Otro elemento desde el cual se representa al indígena desde el mundo enfermero es la higiene. Gran parte de las enfermeras consideran que los indígenas no se bañan, presentan malos olores, permanecen con piojos y se niegan a la orden de bañarse. Esta situación, junto a la dificultad de la práctica del cuidado a estos pacientes, influyen en la experiencia del cuidado a tal punto que algunos evitan la atención o desarrollan una actitud evasiva frente a la situación, sobre todo por la posibilidad de entrar en contacto con los pacientes. Frente a esto la visión del indígena sostiene que deben atravesar largas horas de camino y por lo tanto suda, tienen que exponerse a climas extremos, tienen poca ropa, así como situaciones en las que la intimidad y el significado que le dan al cuerpo, da ejemplo de lo que representa la diversidad cultural.

Otro de los aspectos con los que se asocia la representación del paciente tiene que ver con su identificación del indígena. Generalmente, el personal de enfermería concibe al indígena como uno solo y no distingue la variación cultural que existe entre un grupo y otro. En la Sierra Nevada de Santa Marta, habitan 4 grupos, 3 de los cuales acude a la ciudad para recibir su atención, lo mismo que la comunidad chimila, y en menor proporción integrantes de la comunidad wayuu, yukpa y zenu, quienes habitan ciudades aledañas. En otras palabras, el personal de enfermería no reconoce la diversidad cultural, sino que esencializa su visión a partir de una concepción homogénea de lo indígena. Algunas de las personas entrevistadas indicaron que distinguen uno de otros, pero durante los relatos mencionaron costumbres y denominaciones de uno de estos grupos y lo usaron en otros que no la practican, dando cuenta del poco conocimiento de la diversidad cultural indígena.

Uno de los aspectos determinantes de la representación y con el cual se argumenta la dificultad del cuidado, es el rechazo a los procedimientos o acciones del cuidado. Parte de esta situación se presenta asociada a la idea de "trato especial” con la que en muchas ocasiones 
se concibió el paciente indígena, así como lo “difícil” de la situación. $\mathrm{Al}$ referirse a la experiencia del cuidado al indígena, las enfermeras entrevistadas resaltaron reiterativamente los casos en los que el indígena rechazó los procedimientos, en la mayoría de los casos con el argumento de la cultura indígena, por prohibición o porque los procedimientos tradicionales son mejores. Generalmente el rechazo se daba frente a curaciones, cesáreas, transfusiones sanguíneas, citologías y vacunas, sin embargo, luego de la respectiva explicación, en varios de los casos los pacientes accedían a los procedimientos.

Un último grupo de representaciones giran en torno a la actitud del indígena. En este caso, los testimonios son contradictorios, porque por un lado se presenta la idea de un indígena poco comunicativo y en ocasiones grosero, y por otro, asequible, obediente y sumiso, con una capacidad de espera amplia, opuesto a la dinámica de atención en salud para la población en general, que se presenta más activa en la exigencia de los derechos como paciente. Esta actitud pasiva del indígena, en el contexto de la atención en salud de la ciudad, en ocasiones prolonga su estancia en la institución y aunque nunca se queja, esa experiencia de demora motiva su visión negativa de las instituciones de salud, a las que generalmente ven como un lugar donde pueden morir.

El panorama de las representaciones sobre el paciente indígena, por parte de las enfermeras, da cuenta de las limitaciones para el cuidado en contexto intercultural, sobre todo, porque muestran el poco conocimiento sobre la población indígena y su cultura, así como las imágenes y visiones que pueden estar interviniendo en la experiencia de cuidar. En ese sentido, la asociación del paciente indígena con una situación difícil, demuestran las limitaciones que enfrenta la enfermera en un contexto intercultural.

\section{Comunicación intercultural}

Un segundo aspecto que marca la experiencia enfermera en contexto intercultural es la comunicación. Frente a este caso se establecieron dos niveles que ayudan a comprender el papel de la comunicación. En primer lugar, relacionado con el hecho que, el contexto intercultural samario es plurilingüe, debido a que cada una de las comunidades que reciben la atención en salud en la ciudad posee lengua propia, algunos de los cuales, no dominan el idioma castellano y otros, impulsados por el temor que produce estar en un lugar con el que están poco familiarizados, guarda silencio o evita hablar, lo que lleva a que el personal de la salud interprete que no logran comunicarse. Asimismo, existen otros factores influyentes en el uso del castellano, entre los cuales, se encuentra la responsabilidad asumida por el hombre en el público, lo cual es asociado con machismo o sumisión femenina. El segundo nivel es un poco más complejo, en el cual la problemática va más allá del uso de idiomas diferentes. La primera situación generalmente se resuelve a través de un intérprete, papel que desempeñan los familiares del indígena, una auxiliar de enfermería indígena u otro miembro del equipo de salud de la Institución Prestadora del Servicio de Salud Indígena (IPSI). Sin embargo, la atención en salud en contexto intercultural, así como el cuidado de enfermería se enfrenta al problema de la traducción cultural, es decir, que no solo se limita al reemplazo de una palabra por otra, sino también la visión del mundo, entre los que aparecen aspectos relacionados con la salud y la enfermedad y en aspectos que varían de una cultura a otra, como la intimidad, creencias religiosas, manejo del espacio, entre otras.

\section{Estrategias de cuidado e intervenciones de enfermería}

Pese a las limitaciones existentes manifestadas a partir de las representaciones y la comunicación intercultural, la experiencia del cuidado de enfermería reúne también una variedad de iniciativas que asumen las enfermeras para brindar el cuidado a los pacientes indígenas de manera exitosa. Esta dinámica, generalmente se asocia con la indagación por la cultura del paciente y el interés por descubrir algunos aspectos de los elementos que la conforman. Las enfermeras entrevistadas plantearon este acercamiento como parte de la estrategia de comunicación y mejoramiento de la relación interpersonal con el paciente, descubriendo en muchos casos que entendían en castellano y que poco a poco, lograban 
explicarles los procedimientos y en otros entender por qué se negaban a recibirlo.

El aumento de indígenas que reciben atención en las entidades de salud del sector urbano de la ciudad, poco a poco contribuyó al desarrollo de una experiencia de cuidado de enfermería a comunidades indígenas, cuyo efecto se evidenció entre los diferentes actores. Tanto indígenas como enfermeras y en general los miembros del equipo de salud, se fueron familiarizando con la presencia de indígenas en el área asistencial y a las situaciones ya señaladas. De esta manera, en las últimas dos décadas aproximadamente, el personal de enfermería fue desarrollando de manera empírica intervenciones impulsadas por el deseo de mejorar la salud de los pacientes.

Estas intervenciones varían según la situación de cuidado y el paciente. Muchas de ellas están relacionadas con la educación, con el propósito de "enseñarles cómo deben hacer las cosas para mejorar su salud”. Gran parte de estas intervenciones son en su mayoría etnocéntricas, porque se realizan desde la concepción occidental, pero poco a poco, la experiencia ha permitido ir desarrollando una sensibilidad frente a la cultura del paciente, permitiendo el ejercicio de acciones orientadas desde sus costumbres, que van desde la conservación de aseguranzas $^{2}$ en niños, devolución de la placenta $^{3}$ y rituales realizados por los líderes espirituales de la comunidad.

\section{Algunas reflexiones}

Representaciones, comunicación, estrategias e intervenciones, son solo cuatro de los múltiples aspectos a partir de los cuales se puede abordar las experiencias de enfermería en contexto intercultural y que resultan suficientes para dar cuenta de algunos puntos críticos con los que aún se enfrenta la práctica de enfermería frente a estas situaciones. En primer lugar, por ejemplo, las representaciones sobre el paciente indígena revelan lo mucho que falta para el

\footnotetext{
${ }^{2}$ Hilo de algodón que el mamo o líder espiritual coloca en la muñeca de la persona luego de haber realizado la confesión.

${ }^{3}$ Para los Arhuacos de la SNSM la placenta es la primera madre del indígena y debe ser devuelta a la tierra, como representación del vínculo entre la persona y la naturaleza.
}

reconocimiento del otro y sobre todo de la superación de los estigmas históricos a los que fueron sometidas estas poblaciones y de las cuales depende la calidad del cuidado. En ese sentido, es necesario superar el estereotipo que recae sobre el indígena, concibiéndolo más allá de la concepción salvaje - civilizado. Las estrategias e intervenciones de enfermería realizadas, si bien constituyen un aporte importante a la atención de los pacientes indígenas, es otro de los aspectos que requiere revisión, toda vez que dichas acciones no se realizan fundamentadas en un soporte investigativo que les haya permitido comprender la cultura del paciente, sino por medio de métodos empíricos y desde visiones fundamentadas en el desconocimiento de la cultura indígena $\mathrm{y}$ generalmente orientadas desde imágenes erróneas de sus costumbres. No obstante, esta actitud de las profesionales de enfermería, puede considerarse como una fortaleza para encaminar una experiencia de cuidado orientada desde la enfermería transcultural.

La literatura sobre el tema que ofrece la enfermería sobre el tema es relativamente poca, aunque se evidencian trabajos que empiezan a explorar las situaciones del indígena, resaltando las barreras en la atención (Hautecoeur, Zunzunegui, \& Vissandjee, 2007), así como valoraciones realizadas desde la enfermería transcultural, que empiezan a alimentar el repertorio de estudios que puedan ser utilizados como referencia para un cuidado basado en la cultura (Laza Vásquez \& Cárdenas, 2008; Oviedo, Hernández, Mantilla, Arias, \& Flórez, 2014; Ramos Lafont, 2011). Varios trabajos coinciden en las dificultades del idioma para brindar cuidado (Mendoza, Ximena, Peña, \& Lucía, 2009; Montero, 2011). Por su parte, Rojas (2011, 2012), encuentra en otra ciudad colombiana, similitudes a los hallazgos aquí presentados, relacionados con lo difícil que es para las enfermeras cuidar a pacientes indígenas, así como la comunicación y el etnocentrismo.

En cuanto a las estrategias e intervenciones, las publicaciones registran propuestas orientadas desde enfoques y teorías de enfermería específicas, pero no se hallaron estudios que resaltaran la manera en que estos procedimientos se desarrollen de manera empírica. 


\section{CONCLUSIONES}

Las experiencias de cuidado de enfermería en contextos interculturales dan cuenta de la necesidad de seguir investigando y formando al personal de enfermería desde una visión holística y centrada en el desarrollo de la competencia cultural. Además, requiere del desarrollo de iniciativas para que el personal de salud que labora en la actualidad tenga acceso al conocimiento científico que circula sobre las comunidades indígenas, desarrollando con esto la sensibilidad necesaria y el fundamento conceptual que permita superar el etnocentrismo. De igual forma, diseñar estrategias de intervención orientadas desde las teorías y principios de las cuales dispone la enfermería, con el fin de generar estrategias que disminuyan las desigualdades en los procesos de atención en salud que presentan condiciones de interculturalidad.

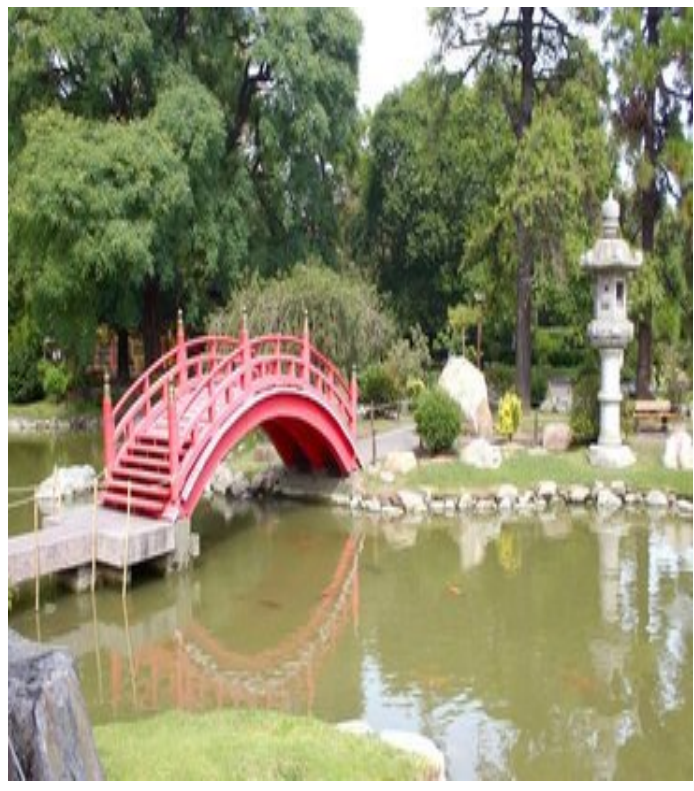

Fuente:

https://es.freeimages.com/search/culture?free=1

\section{BIBLIOGRAFÍA}

Casasa, P., Ramírez, A., Sánchez, A., \& Campos, G. (2010). Enfermería cultural: Antropoenfermería, Salud, migración y multiculturalidad en América Latina. México: Miguel Ángel Porrúa.

Guber, R. (2011). La etnografía. Método, campo y reflexividad. Buenos Aires: Siglo XXI.

Hall, S. (2010). El trabajo de la representación. En E. Restrepo \& K. Walsh (Eds.), Sin garantías. Trayectorias y problemáticas en estudios culturales. (pp. 447-482). Popayán Colombia, Lima: Envíon Editores. Instituto de Estudios Peruanos, Instituto Pensar.

Hammersley, M., \& Atkinson, P. (2004). Etnografía. Métodos de investigación. Barcelona: Paidós Básica.

Laza Vásquez, C., \& Cárdenas, F. J. (2008). Una mirada al cuidado en la gestación desde la enfermería transcultural. Revista Cubana de Enfermería, 24(3-4), 0-0. Recuperado de: http://scielo.sld.cu/scielo.php?script=sci_arttext \&pid=S0864-03192008000300009

Leininger, M., \& McFarland, M. (2002). Transcultural nursing: concepts, theory, research and practice. Chicago: McGraw-Hill. Lillo Crespo, M., Vizcaya Moreno, F., Santamaría, D., Mario, J., \& Galao Malo, R. (2004). Investigación cualitativa en enfermería y competencia cultural. Index de Enfermería, 13(44-45), 47-50. Recuperado de http://scielo.isciii.es/scielo.php?script=sci_arttex t\&pid=S1132-12962004000100010

McFarland, M. (2011). Madeleine M. Leininger: Teoría de la Diversidad y de la Universalidad de los cuidados culturales. En M. Alligood \& A. Marriner (Eds.), Modelos y teorías en Enfermería. Barcelona: Elsevier. 454-479.

Mendoza, I., Ximena, T., Peña, N., \& Lucía, A. (2009). Reflexiones sobre la práctica enfermera: una aproximación teórica-vivencial desde la perspectiva de la interacción intercultural. Index de Enfermería, 18(2), 116-120. Recuperado de http://scielo.isciii.es/scielo.php?script=sci_arttex t\&pid=S1132-12962009000200010

Montero Mendoza, E. (2011). Percepción de los habitantes indígenas de áreas rurales respecto al primer nivel de atención médica: El caso del sureste de Veracruz, México. Salud colectiva, 7(1), 73-86. Recuperado de: http://www.scielo.org.ar/scielo.php?script=sci_a rttext\&pid=S1851-82652011000100006 
Oviedo, M., Hernández, A., Mantilla, B., Arias, K., \& Flórez, N. (2014). Comunidades Embera y Wounaan del departamento de Chocó: prácticas de cuidado durante el proceso de gestación. Revista de la Facultad Nacional de Salud Pública, 32(2). Recuperado de http://aprendeenlinea.udea.edu.co/revistas/index. php/fnsp/article/view/16026

Poveda, A. (2001). ¿Antropoenfermería o enfermeantropología? Cultura de los Cuidados, 5 (9), 5 - 9. Recuperado de https://rua.ua.es/dspace/bitstream/10045/4942/1/ CC_09_01.pdf

Purnell, L. D. (1999). El modelo de competencia cultural de Purnell: descripción y uso en la práctica, educación, administración e investigación. Cultura de los cuidados, 3(6), 91$102 . \quad$ Recuperado

de

http://rua.ua.es/dspace/handle/10045/5157

Quijano, A. (2014). Colonialidad del poder y clasificación social. En CLACSO (Ed.), Cuestiones y horizontes: de la dependencia histórico-estructural $a$ colonialidad/descolonialidad del poder. Buenos Aires: CLACSO. Recuperado de http://biblioteca.clacso.edu.ar/clacso/se/2014050 6032333/eje1-7.pdf
Ramos, C. P. (2011). Prácticas culturales de cuidado de gestantes indígenas que viven en el Resguardo Zenú ubicado en la Sabana de Córdoba. (Tesis de Maestría no publicada), Facultad de Enfermería. Universidad Nacional de Colombia, Bogotá. Recuperado de http://www.bdigital.unal.edu.co/5416/

Rojas, J. G. (2011). Significado de la experiencia de cuidar a pacientes indígenas en las salas de hospitalización del Departamento de Antioquia-Colombia. Tesis de Doctorado no publicada), Universidad de Alicante: Alicante. Recuperado de https://rua.ua.es/dspace/bitstream/10045/24158/1 /Juan_Guillermo_Rojas.pdf Sarrazin, J. P. (2015). Representaciones sobre lo indígena y su vínculo con tendencias culturales globalizadas. Anagramas -Rumbos y sentidos de la comunicación, 14(27), 163-183.

Siles González, J., Cibanal Juan, L., VizcayaMoreno, M. F., Bravo, G., María, E., Santamaría, D. García Hernández, E. (2001). Una mirada a la situación científica de dos especialidades esenciales de la enfermería contemporánea: la antropología de los cuidados y la enfermería transcultural. Cultura de los Cuidados, 1 (10). 72-87. Recuperado de http://rua.ua.es/dspace/handle/10045/4938 